NM-JP-7

\title{
Homogenization analysis of plastic size effects using discrete dislocation plasticity approach*
}

\author{
Dai Okumura** and Nobutada Ohno** \\ **Department of Mechanical Science and Engineering, Nagoya University \\ Furo-cho, Chikusa-ku, Nagoya, Japan \\ E-mail:okumura@mech.nagoya-u.ac.jp
}

\begin{abstract}
Homogenization analysis of lamellar composites consisting of elastic and elastic-plastic layers is performed using a discrete dislocation plasticity approach. Plastic size effects are caused by dislocation pile-ups at interfaces. It is shown that the strain hardening in elastic-plastic layers arises depending on the thickness of elastic layers as well as the gap between slip planes in adjacent elastic-plastic layers. In a case where the thickness of elastic layers is several dozen $\mathrm{nm}$, the strain hardening in elastic-plastic layers reduces as the gap of the slip planes decreases. It is found that this effect is attributed to the long range stress due to the pile-ups in adjacent elastic-plastic layers.
\end{abstract}

Key words: Plasticity, Dislocations, Size effects, Homogenization

\section{Introduction}

Plastic size effects observed in polycrystals and metallic composites with submicron structures are attributed to dislocation pile-ups at interfaces [1]. The pile-ups contribute to increasing dislocation densities near interfaces and to generating elastic long range stresses. The long range stresses are considered not only to work as back stresses in the domain including the pile-ups but also to affect adjacent domains through interfaces. When lamellar composites consist of elastic and elastic-plastic layers, the plastic deformation is apt to induce the pile-ups of oppositely signed dislocations at the interfaces of both sides of the elastic layer (see Fig. 1). The long range effects due to signed and oppositely signed dislocations act to weaken each other as the thickness of the elastic layer decreases. Consequently, the plastic size effects of lamellar composites appear to arise, depending to a large degree, on the thickness of the elastic layer. Discrete dislocation plasticity simulations [e.g., 2-5] are considered to have the ability to predict the above plastic size effects. Because the long range interactions of dislocations are directly implemented as their elastic fields into the frameworks.

In this study, the plastic size effect analysis of lamellar composites is performed using the approach based on discrete dislocation plasticity and periodic homogenization. Section 2 is devoted to a brief outline of the discrete dislocation plasticity approach developed by the present authors [5]. It is shown that micro- and macro-stresses are expressed using the solutions obtained by solving two periodic boundary value problems specialized for the representative volume element analysis of periodic composites. Section 3 gives a model lamellar composite consisting of elastic and elastic-plastic layers. In Section 4, the numerical results are shown to discuss the plastic size effects depending on the thickness of elastic layers and the gap between slip planes in adjacent elastic-plastic layers. Finally, Section 5 presents a summary.

\section{Theory}

This section is devoted to a brief outline of the discrete dislocation plasticity approach, which has been developed by the present authors [5], and is based on the superposition method developed by Van der Giessen and Needleman [4] and on the decomposition of displacements into macro and perturbed components.

Figure 1 illustrates the problem analyzed using the present discrete dislocation plasticity approach. Periodic lamellar composites are composed of elastic and elastic-plastic layers, and are subjected to macroscopic uniform deformation 
described by macroscopic stress $\Sigma_{i j}$ and/or macroscopic strain $E_{i j}$. The lamellar composites are defined by infinitely periodic arrangements of a periodic unit $V$. Elastic and elastic-plastic layers are generally regarded as inclusions $V_{1}$ and matrices $V_{\mathrm{M}}$, respectively, which are assumed to be isotropic elastic materials, but the motion of individual dislocations are allowed in the matrix area.

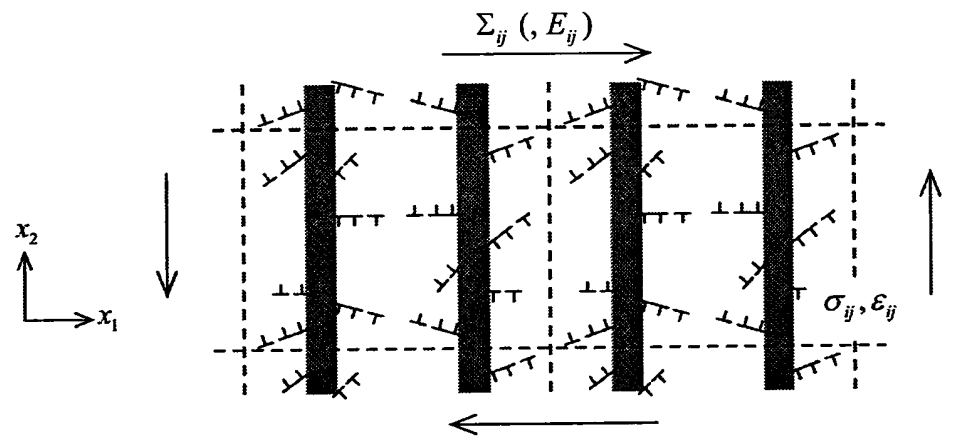

Fig.1. Periodic lamellar composites subjected to macroscopic uniform deformation described by macroscopic stress $\Sigma_{i j}$ (and/or macroscopic strain $E_{i j}$ ), and periodic unit $V$ (each area surrounded by broken lines) consisting of elastic layers (regarded as inclusions $V_{\mathrm{I}}$, gray areas) and elastic-plastic layers (regarded as matrices $V_{\mathrm{M}}$, white areas) that allow individual dislocation motions.

Since the approach used here is based on the superposition method, the above-mentioned problem is divided into two problems: the dislocation problem and the complementary problem [4,5]. The dislocation problem gives the solution of self-equilibrated fields for dislocations in the infinite medium of the homogeneous matrix, while the complementary problem gives the solution of fields to correct for the presence of inclusions as well as the macroscopic uniform deformation. The summation of two problems leads to the solution of the original problem.

When the dislocation problem is solved by superposing analytical solutions for individual dislocations under self-equilibrium in the infinitely large medium [1], the micro strain field $\tilde{\varepsilon}_{i j}$ for the dislocation problem is only used to solve the complementary problem in the present approach [5]. Consequently, micro and macro stresses are expressed as

$$
\begin{aligned}
& \sigma_{i j}=\left(c_{i j k l}+c_{i j p q} \chi_{p, q}^{k l}\right)\left(E_{k l}-E_{k l}^{\mathrm{p}}\right)+c_{i j k l}\left(\tilde{\varepsilon}_{k l}+\varphi_{k, l}\right) \\
& \Sigma_{i j}=C_{i j k l}^{\mathrm{H}}\left(E_{k l}-E_{k l}^{\mathrm{p}}\right)+\Sigma_{i j}^{\mathrm{D}}
\end{aligned}
$$

where $C_{i j k l}^{\mathbf{H}}$ and $\Sigma_{i j}^{\mathbf{D}}$ are given, respectively, by

$$
\begin{aligned}
& C_{i j k l}^{\mathrm{H}}=\frac{1}{V} \int_{V}\left(c_{i j k l}+c_{i j p q} \chi_{p, q}^{k l}\right) d V \\
& \Sigma_{i j}^{\mathrm{D}}=\frac{1}{V}\left\lceil c_{i j k l}\left(\tilde{\varepsilon}_{k l}+\varphi_{k, l}\right) d V\right.
\end{aligned}
$$

Here $c_{i j k l}$ is the elastic stiffness, which characterizes the elastic properties of the matrix and the inclusion, that is

$$
c_{i j k l}= \begin{cases}c_{i j k l}^{\mathrm{M}} & \text { in } V_{\mathrm{M}} \\ c_{i j k l}^{\mathrm{I}} & \text { in } V_{1}\end{cases}
$$

The tensor $E_{i j}^{\mathrm{p}}$ is the macroscopic plastic strain due to the motion of individual dislocations, and is incrementally calculated using its rate form

$$
\dot{E}_{i j}^{\mathrm{p}}=\frac{1}{2 V} \sum_{\alpha=1}^{N}\left(\int_{l}(\alpha) v^{(\alpha)} d l^{(\alpha)}\right)\left(b_{i}^{(\alpha)} m_{j}^{(\alpha)}+m_{i}^{(\alpha)} b_{j}^{(\alpha)}\right)
$$

where $l^{(\alpha)}$ and $v^{(\alpha)}$ are the line of dislocation $\alpha(\alpha=1,2,3, \ldots N)$ and its velocity. The individual dislocations belong to a slip system, in which $b_{i}^{(\alpha)}$ and $m_{i}^{(\alpha)}$ are Burgers' vector and the unit vector normal to the glide plane, respectively. Moreover, $\chi_{i}^{k l}$ and $\varphi_{i}$ are perturbed components of displacements, and are periodic. The solutions $\chi_{i}^{k l}$ and $\varphi_{i}$ are obtained by computationally solving two periodic boundary value problems

$$
\begin{aligned}
& \int_{V} \delta u_{i, j}^{*} c_{i j k l} \chi_{k, l}^{p q} d V=-\int_{V} \delta u_{i, j}^{*} c_{i j p q} d V \\
& \int_{V} \delta u_{i, j}^{*} c_{i j k l} \varphi_{k, l} d V=-\int_{V} \delta u_{i, j}^{*}\left(c_{i j k l}-c_{i j k l}^{\mathrm{M}}\right) \tilde{\varepsilon}_{k l} d V
\end{aligned}
$$

where $\delta u_{i}^{*}$ is an arbitrary periodic variation. The solution $\chi_{i}^{k l}$ arises depending on the presence of inclusions, and 
independent of the dislocation problem. By contrast, the solution $\varphi_{i}$ arises depending on the elastic fields of the dislocation problem. Therefore, the second term on the right hand side of micro stress expression (1) is the stress field induced by dislocations in composites. In Section 4, this term $\sigma_{i j}^{\mathrm{d}}=c_{i j k l}\left(\tilde{\varepsilon}_{k l}+\varphi_{k, l}\right)$ is regarded as the long range stress of dislocations.

The incremental analysis is performed using Eqs. (1)-(8). At each incremental step, the dislocation velocity, and the generations and annihilations of dislocations are analyzed based on constitutive equations [2-5]. The detailed procedure can be found in [5].

\section{Analytical model}

Figure 2 shows the periodic unit of a model lamellar composite, which are analyzed to investigate the plastic size effects due to dislocation pile-ups at interfaces. Each periodic unit is composed of elastic-plastic layers of thickness $400 \mathrm{~nm}$ and elastic layers of thickness $w_{\mathrm{I}}=40$ or $400 \mathrm{~nm}$. The Young's modulus of the elastic-plastic layer is taken as $E_{\mathrm{M}}=70 \mathrm{GPa}$, whereas the Young's modulus of the elastic layer is $E_{\mathrm{I}}=3 E_{\mathrm{M}}$. The Poisson ratio of both layers is identically $v_{\mathrm{M}}=v_{\mathrm{I}}=0.33$. Each elastic-plastic layer has one dislocation source, located at points $\mathrm{X}$ and $\mathrm{Y}$ in Fig. 2. The gap between the slip planes on the sources is taken as $H=0,100$ and $200 \mathrm{~nm}$. Moreover, the periodic unit does not contain initial dislocations, and is subjected to macroscopically in-plane pure shear. The loading condition is expressed as $\dot{E}_{12}=500 \sec ^{-1}$ and $\Sigma_{11}=\Sigma_{22}=0$, and the time increment used here is $\Delta t=0.05 \mathrm{nsec}$.

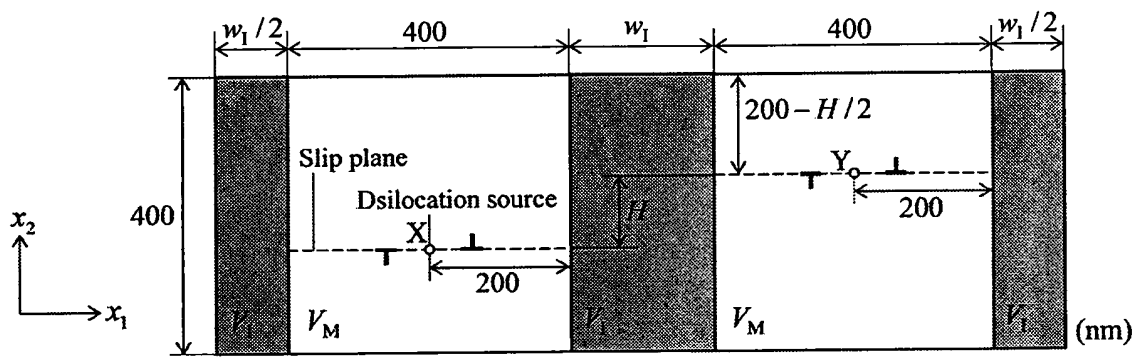

Fig. 2. Periodic unit with dislocation sources at points $\mathrm{X}$ and $\mathrm{Y}$; the slip plane gap $H$ is taken as 0,100 , and $200 \mathrm{~nm}$, and the elastic layer thickness $w_{\mathrm{I}}$ is taken as 40 and $400 \mathrm{~nm}$.

When the magnitude of the shear stress at the source, $\tau=\left|\sigma_{12}\right|$, has exceeded the critical stress $\tau_{\text {nuc }}$ during a period of time $t_{\text {nuc }}$, the dislocation sources emit a pair of positive and negative edge dislocations, which have Burgers vector $b=0.25$ $\mathrm{nm}$ in the horizontal direction, with the horizontal gliding plane, and with the dislocation lines perpendicular to the plane. The distance between the two dislocations emitted from the dislocation source is determined to balance the shear stress $\tau$ and the attractive force between the two dislocations. The motion of each dislocation is controlled by the in-plane component of the Peach-Koehler force $f$. The in-plane force, $f$, and the velocity, $v$, of the dislocation in the direction of the Burgers vector are related by the linear relation $v=f / B$, where $B$ is the drag coefficient. In this analysis, the values $\tau_{\text {nuc }}=49 \mathrm{MPa}, t_{\text {nuc }}=10$ nsec, and $B=0.1 \mathrm{MPa}$ nsec are used [4,5].

Each periodic unit has a rectangular domain. Hence, in the dislocation problem, the strain field $\tilde{\varepsilon}_{k l}$ is calculated by applying the exact solutions to an infinitely long string of edge dislocations at a mutual distance in the horizontal direction, and superposing the fields due to the strings periodically arranged in the vertical direction with a cut-off cell size [4,5]. Four-noded isoparametric elements are used to discretize the individual periodic units, and the number of the elements in the periodic units, which depends on the elastic layer thickness $W_{1}$ and the slip plane gap $H$, is between 11,520 and 18,480. The minimum element edge length around the interfaces, where dislocation pile-ups are expected, is $1 \mathrm{~nm}$.

\section{Results and discussion}

Figures 3(a) and 3(b) show the macroscopic stress - plastic strain responses for the elastic layer of thickness $w_{\mathrm{I}}=40 \mathrm{~nm}$ and $400 \mathrm{~nm}$, respectively. Here $\Sigma_{12(\mathrm{M})}$ is the macroscopic shear stress in the elastic-plastic layer, and is defined as $\Sigma_{12(\mathrm{M})}=\left(1 / V_{\mathrm{M}}\right) \int_{1 / \mathrm{M}} \sigma_{12} d V$, while $E_{12(\mathrm{M})}^{\mathrm{p}}$ is the macroscopic plastic shear strain in the elastic-plastic layer, and is defined as $E_{12(\mathrm{M})}^{\mathrm{p}}=\left(V / V_{\mathrm{M}}\right) E_{12}^{\mathrm{p}}$. These jagged responses in Fig. 3 occur for two reasons; a negative linear decrease during periods in which emitted dislocations move, and a positive sharp increase after dislocations have piled up at the interfaces. Strain hardening in the elastic-plastic layer arises depending on the thickness of the elastic layer as well as the gap between the slip planes. Fig. 3 shows that the results for $w_{\mathrm{I}}=400 \mathrm{~nm}$ are independent of the variations of the gap $H$, whereas the results for $w_{\mathrm{I}}$ 
$=40 \mathrm{~nm}$ are considerably dependent on the variations of the gap $H$. In the case of $w_{1}=40 \mathrm{~nm}$, strain hardening in the elastic-plastic layer decreases as the gap decreases from $200 \mathrm{~nm}$ to $0 \mathrm{~nm}$. This remarkable effect on the strain hardening that occurs only in the case of $w_{\mathrm{I}}=40 \mathrm{~nm}$, can be regarded as the plastic size effect caused by decreasing the thickness of elastic layers.
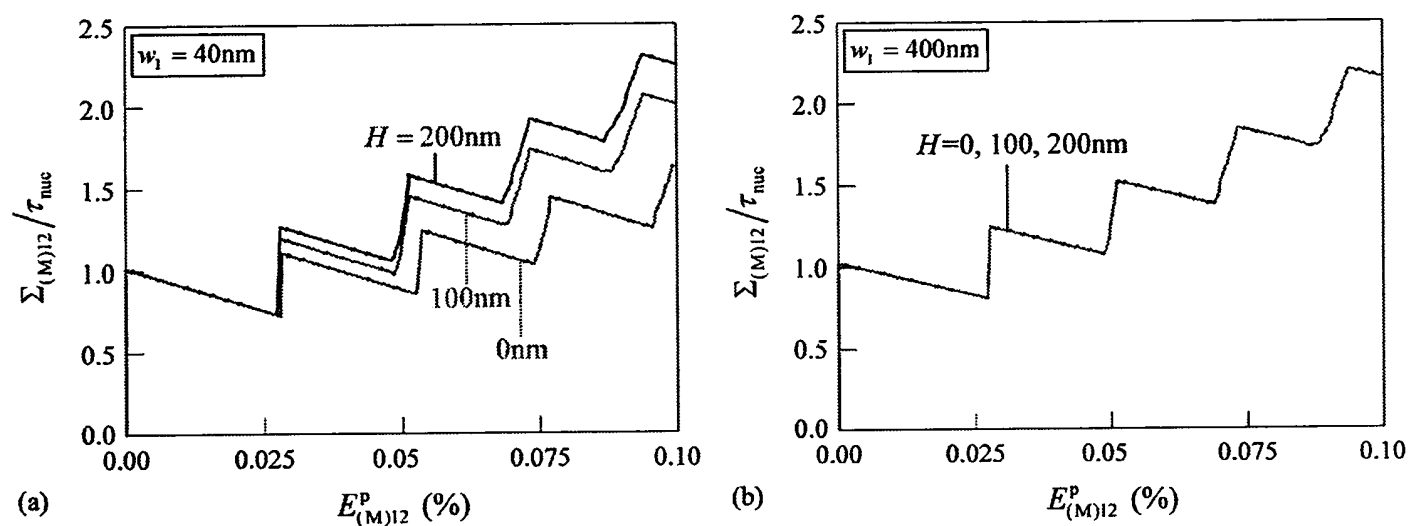

Fig. 3. Macroscopic shear stress - macroscopic plastic shear strain relations averaged in elastic-plastic layers; (a) for $w_{\mathrm{I}}=40$ $\mathrm{nm}$, and (b) for $w_{\mathrm{I}}=400 \mathrm{~nm}$.
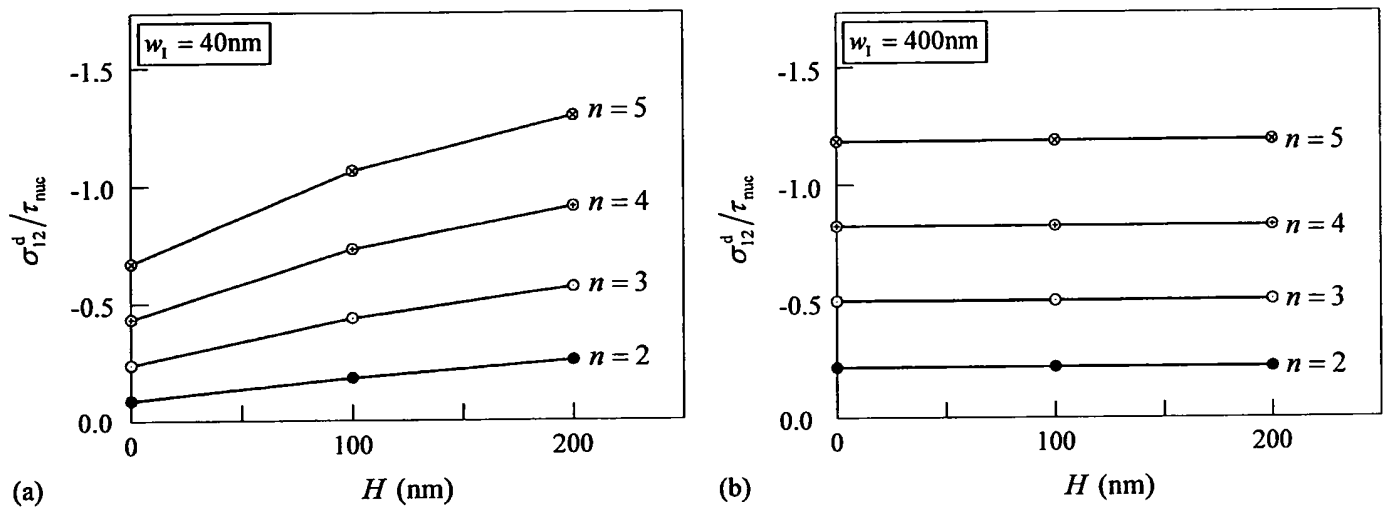

Fig. 4. Shear component of the long range stress, $\sigma_{12}^{\mathrm{d}}=c_{12 k l}\left(\tilde{\varepsilon}_{k l}+\varphi_{k, l}\right)$, at dislocation sources just before emitting the $n$-th dislocation from dislocation sources; (a) for $w_{\mathrm{I}}=40 \mathrm{~nm}$, and (b) for $w_{\mathrm{I}}=400 \mathrm{~nm}$.

To analyze these responses from the microscopic point of view, Fig. 4 indicates the shear component of the long range stress due to dislocations, at dislocation sources, just before emitting the $n$-th dislocation from each dislocation source. Hence $\sigma_{12}^{\mathrm{d}}$ is defined as $\sigma_{12}^{\mathrm{d}}=c_{12 k l}\left(\tilde{\varepsilon}_{k l}+\varphi_{k, l}\right)$, and can therefore be considered as the back stress due to piled-up dislocations. The back stress is well-known to work at preventing the activation of dislocation sources as well as the motion of dislocations. It is found from Figs. 3 and 4 that an increase in the back stress causes an increase in the strain hardening in the elastic-plastic layer. In the case of $w_{\mathrm{I}}=40 \mathrm{~nm}$, the back stress increases as the gap increases, while, in the case of $w_{\mathrm{I}}=400 \mathrm{~nm}$, the increase in the back stress is independent of the gap variation.

To explain the mechanism of the above mentioned plastic size effect, we refer to Fig. 5 which indicates the distributions of $\sigma_{12}^{d}$ in the periodic unit just before emitting the 4th dislocation from dislocation sources. It can clearly be seen from Fig. 5 that the long range stress, due to dislocation pile-ups, gives two main effects especially in the direction of the pile-ups; they are the back stresses behind the pile-ups and the positive stresses ahead of the pile-ups. Figs. $5(\mathrm{~d})$-(f) show that the elastic layer of thickness $400 \mathrm{~nm}$ prevents the positive stresses from affecting the adjacent elastic-plastic layer. The distributions in the elastic-plastic layers for $H=0,100$, and $200 \mathrm{~nm}$ are almost the same because of considering the periodic arrangements of periodic units. Consequently, the macroscopic and microscopic responses for $w_{\mathrm{l}}=400 \mathrm{~nm}$ (see Figs. 3(b) and 4(b)) are independent of the gap variations. By contrast, Figs. 5(a)-5(c) show that the elastic layer of thickness $40 \mathrm{~nm}$ enables the positive stresses to affect the adjacent elastic-plastic layer. In the case of $H=200 \mathrm{~nm}$, the long range stress acting through the elastic layer contributes to increasing the stress level in the elastic-plastic layer, but does not influence the dislocation source 
due to the directional characteristics of the long range stress (see Fig. 5(c)). However, it is apparent from Fig. 5(a) that the gap of $0 \mathrm{~nm}$ enables the long range stress induced by the pile-ups in the adjacent elastic-plastic layer to weaken the back stress generated by the pile-ups in the corresponding elastic-plastic layer. Therefore, the elastic layer of thickness $40 \mathrm{~nm}$ decreases the back stress at dislocation sources and the macroscopic strain hardening as the gap decreases from $200 \mathrm{~nm}$ to $0 \mathrm{~nm}$.

(a)

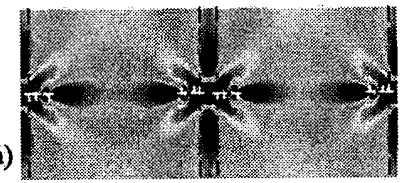

(b)

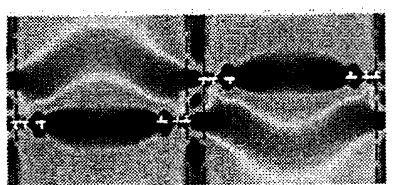

(c)

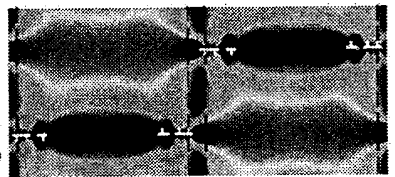

(d)

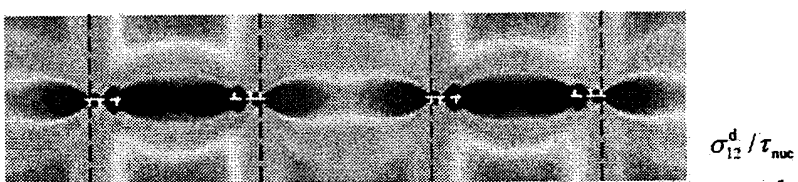

(e)

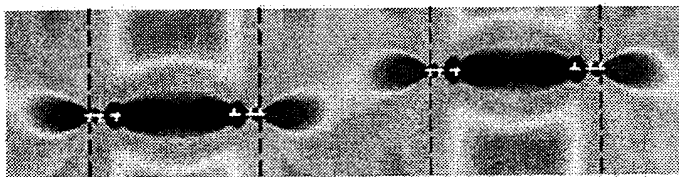

(f)

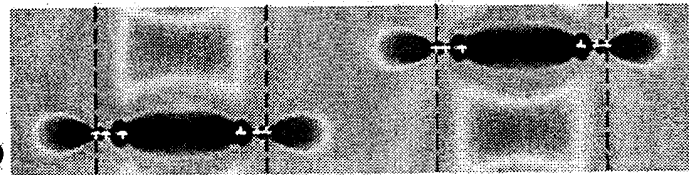

0

Fig. 5. Distributions of the long range stress $\sigma_{12}^{\mathrm{d}}$ just before emitting the 4th dislocation from dislocation sources; (a) for $w_{1}$ $=40 \mathrm{~nm}$ and $H=0 \mathrm{~nm}$, (b) for $w_{\mathrm{I}}=40 \mathrm{~nm}$ and $H=100 \mathrm{~nm}$, (c) for $w_{\mathrm{I}}=40 \mathrm{~nm}$ and $H=200 \mathrm{~nm}$, (d) for $w_{\mathrm{I}}=400 \mathrm{~nm}$ and $H=$ $0 \mathrm{~nm},(\mathrm{e})$ for $w_{\mathrm{l}}=400 \mathrm{~nm}$ and $H=100 \mathrm{~nm}$, and (f) for $w_{\mathrm{I}}=400 \mathrm{~nm}$ and $H=200 \mathrm{~nm}$.

\section{Summary}

In this study, a model lamellar composite was analyzed using the discrete dislocation plasticity approach developed by the present authors [5]. It was shown that the strain hardening in elastic-plastic layers arises depending on the thickness of elastic layers as well as on the gap between slip planes in adjacent elastic-plastic layers. It was also revealed that elastic layers with less than submicron thickness decreases the strain hardening in elastic-plastic layers as the gap of the slip planes decreases. It was found that the long range stress due to the dislocation pile-ups in adjacent elastic-plastic layers drastically weakens the back stress induced by the dislocation pile-ups in the corresponding elastic-plastic layer when the thickness of elastic layers is less than submicron.

Finally, it should be noted that the present study was performed using the model lamellar composites that do not contain multiple slip systems, elastic anisotropy, threading dislocations and so on. More realistic models should be employed for advancing understanding of plastic size effects of lamellar composites and for comparing experiments and simulations.

\section{References}

[1] J.P. Hirth, J. Lothe, Theory of dislocations. 2nd ed., John Wiley \& Sons, New York (1982).

[2] A.N. Gulluoglu, D.J. Srolovitz, R. LeSar, P.S. Lomdahl, Scripta Metall. 23, pp.1347-1352 (1989).

[3] H.M. Zbib, M. Rhee, J.P. Hirth, Int. J. Mech. Sci. 40, pp.113-127 (1998).

[4] E. van der Giessen, A. Needleman. Model. Simul. Mat. Sci. Eng. 3, pp.689-735 (1995).

[5] D. Okumura, N. Ohno, K. Yamaguchi, Int. J. Plasticity 27, pp.2040-2055 (2011). 